\title{
Computed tomography imaging findings in hospitalized patients with coronavirus disease 2019 (COVID-19): a descriptive study of 81 cases
}

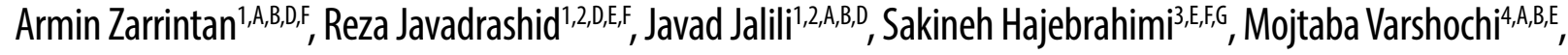 \\ Parisa Hajalioghli1,2,A,E,F, Batool Seifi, ${ }^{1, E, F}$, Mohammad Mirza-Aghazadeh-Attari ${ }^{1,2, A, B, C, D, E, F,}$ \\ Mohammad Kazem Tarzamni ${ }^{1,2, a, B, E}$
}

'Department of Radiology, Faculty of Medicine, Tabriz University of Medical Sciences, Tabriz, Iran ${ }^{2}$ Medical Radiation Sciences Research Group, Department of Radiology, Faculty of Medicine, Tabriz University of Medical Sciences, Tabriz, Iran ${ }^{3}$ Evidenced based Medicine Research Center, Tabriz University of Medical Sciences, Tabriz, Iran

${ }^{4}$ Department of Infectious Disease, Imam Reza Hospital, Tabriz University of Medical Sciences, Tabriz, Iran

\section{Abstract}

\begin{abstract}
Purpose: Severe acute respiratory syndrome-coronavirus-2 (SARS-CoV-2) has proven to be a diagnostic challenge. Early studies have shown that computed tomography (CT) imaging may be useful in diagnosis of these patients. We aim to report CT findings in a series of hospitalized patients.

Material and methods: A total of 81 patients were included in this study. All of the patients were hospitalized and had SARS-CoV-2 infection proven by molecular assay. All patients had a CT scan on the first day of admission. Imaging results were reviewed by two separate radiologists, and imaging findings were documented.

Results: Seventy-eight patients had abnormal CT imaging, while 3 had normal CT imaging. The sensitivity of CT in diagnosing coronavirus disease 2019 (COVID-19) was estimated to be $96 \%$. The most common imaging finding was ground glass opacities, followed by septal thickening. Most lesions were located at the periphery and posterior of the lungs. Most lesions were multifocal, and involved the right lower lobe more frequently. Chest X-rays were normal in 38 patients, and the sensitivity of chest X-ray in diagnosing SARS-Cov-2 was $54 \%$.
\end{abstract}

Conclusions: CT scans could be used in diagnosis of patients with a high sensitivity (93\%). No common imaging findings may also be seen alongside ground glass opacities, based on the degree of disease progression.

Key words: COVID-19, CT scan, virus, epidemic.

\section{Introduction}

In December 2019 a new coronavirus emerged from the Chinese city of Wuhan, which in most cases only caused mild disease, but in some, especially in subjects with preexisting conditions such as hypertension and diabetes, caused severe viral pneumonia, characterized by prominent lower respiratory tract symptoms and a systemic inflammatory response to the virus [1]. Currently, the virus is disseminated in more than 100 countries, and has caused more than a million cases of infection and at least 50000 deaths [2]. The virus has a reproduction number of 2-3, meaning it can easily infect individuals, and has shown to be contagious even when a subject is asymptomatic. Because of this, health systems worldwide have focused on the strategy of early detection and isolation of cases [3]. This in fact has proven to be a major challenge, as a significant portion of those infected do not show symptoms and do not seek medical attention [4]. Another challenge is diagnosing the virus. Currently, accepted au-

Correspondence address:

Mohammad Kazem Tarzamni, Department of Radiology, Faculty of Medicine, Tabriz University of Medical Sciences, Daneshgah Avenu, Tabriz, Iran, e-mail:m.aghazadeh96@gmail.com 
thorities put emphasis on the necessity of frequent and wide scale testing, which is done by molecular assays from samples taken from the upper respiratory tract [5]. Countries with underdeveloped health systems and weak disease surveillance have failed to accommodate the necessary infrastructure needed for such testing. A combination of these two factors has led to undertreatment of patients infected with the virus, especially in subjects who are hospitalized. As early as January 2020, suggestions were made regarding the possible role of computed tomography (CT) imaging in the diagnosis of COVID-19 [6]. Early studies conducted in this regard pointed out the possible benefit of CT imaging [7]. Most of the initial studies came from Korea and China, with limited evidence being presented from elsewhere. In this study we analyze the imaging findings of patients infected with SARS-CoV-2. We also define the sensitivity of CT imaging in the diagnosis of the disease.

\section{Material and methods}

\section{Patients}

In this prospective study all of the patients who were referred to and hospitalized in a tertiary medical center affiliated with a medical sciences university between February $1^{\text {st }} 2020$ and March $29^{\text {th }} 2020$ were included. The aforementioned center was the referral center for infectious disease for a population of 6 million, and patients received medical services from trained specialists. Inclusion criteria consisted of those with a positive polymerase chain reaction test for COVID-19, being hospitalized in an infectious disease ward or intensive care unit and willingness to participate in the study. Exclusion criteria consisted of those with concomitant viral or bacterial pneumonia (diagnosed by clinical assessments or samples from the respiratory tract), and pre-existing lung disease, such as bronchiectasis, cystic fibrosis, pulmonary idiopathic fibrosis, emphysema and silicosis.

\section{Computed tomography imaging protocol}

Subjects were referred to the imaging department based on the algorithm suggested by evidence presented by the World Health Organization (WHO) [8]. All patients had CT imaging with Siemens SOMATOM (Hannover, Germany) and Toshiba Alexion (Kyoto, Japan) machines based on the following technical specifications: axial and sagittal images, automatic tube current modulation with a voltage of $120 \mathrm{kVp}$, matrix size of $512 \times 512$ and $256 \times 256$, increment and thickness of 1.5-2 mm. Analysis of the CT imaging and subsequent staging was done by two separate radiologists with at least 20 years of experience in thoracic imaging, and was done according to the latest checklist provided by the Radiological Society of North America and by Kanne et al. $[9,10]$. The checklist was modified based on the most recent imaging guideline issued by the United states Board of Diagnostic Radiology [11]. These guidelines classify imaging findings into three types (suggestive of COVID-19, inconsistent with COVID-19, and undetermined findings). Staging of radiologic imaging was done according to recommendations from Jin et al. [12]. In this staging, based on disease progression, 5 stages are determined: the ultra-early stage (stage 1) characterized by focal ground glass opacities, the early stage (stage 2 ) characterized by more disseminated ground glass opacities and thickened interlobular septa, the rapid progression stage (stage 3), characterized by large consolidation and air bronchogram, the consolidation stage (stage 4) characterized by multiple patchy consolidations, and the dissipation stage (stage 5) characterized by abiding imaging findings, few patchy consolidations and interlobular septa thickening.

Radiologists were not aware of the results of molecular assays prior to reporting of radiological images.

Radiologists were also not aware of chest X-ray findings of each CT image prior to interpretation of CT imaging. The radiologists reviewed the images separately, and in case of disagreement, a third radiologist with 14 years of experience reviewed the images and a consensus was reached.

\section{Molecular assay}

Samples were taken from the oropharynx and nasopharynx, with their specimens being processed based on the guideline issued by the WHO, with primers approved by the Chinese Center for Disease Control, targeting the $\mathrm{N}$ gene (sequence GAGGAACGAGAAGAGGCTTG 600-800 nM) per reaction. Storage, collection and shipment of all specimens were done according to interim guidance issued by the WHO on March $19^{\text {th }} 2020$ [13].

\section{Statistical analysis}

Microsoft Excel was used for data collection and production of tables. Statistical analysis was done with SPSS, version 19.10. Continuous variables were directly expressed as a range and categorical variables were expressed as numbers and percentages.

\section{Ethical considerations}

This study was approved by the local ethics committee of the medical sciences university where it was performed (Ethical code: IR.TBZMED.REC.1398.1275). All of the patients included in the study had signed pre-designed inform consent notes. Consent for publication was obtained from all of the patients. Identifying information was not presented in any way and data were not shared with any third party. The study was in in compliance with the Declaration of Helsinki. 


\section{Results}

A total of 81 patients were included in our study. All of the patients had tested positive for SARS-CoV-2 prior to inclusion. Forty-one patients were male and 31 were female. The most common age group was 60-70 years. The mean age of patients was $58 \pm 12.1$. Table 1 summarizes the demographic information of patients.

Of all the patients 3 had normal CT imaging. 79 had abnormal imaging. Patients were classified in 5 stages based on lung involvement. The most common imaging group was group 2 with 25 patients and group 3 with 19 patients. The most common imaging finding was ground glass opacity, with 76 patients having it (93.8\%). Most patients had bilateral involvement (66-81.4\%). The most common pattern of involvement was multifocal (61-75.3\%), followed by diffuse involvement (11-13.5\%). The peripheral regions of the lungs were involved in 69 patients $(85.1 \%)$, followed by the posterior regions (54-66.6\%). The most common lobe involved was the right lower (65-80.2\%), followed by the left upper lobe and right upper and middle lobe.

Table 1. Demographic information of the patients included in the study. Data represented as frequency (percentage)

\begin{tabular}{l} 
Parameter \\
\begin{tabular}{|l|c|}
\hline Sex & $n(\%)$ \\
\hline Male & $42(51.8)$ \\
\hline Female & $39(48.1)$ \\
\hline Age & $1(1.2)$ \\
\hline$<10$ & 0 \\
\hline $10-20$ & $2(2.4)$ \\
\hline $20-30$ & $13(16.0)$ \\
\hline $30-40$ & $14(17.2)$ \\
\hline $40-50$ & $11(13.5)$ \\
\hline $50-60$ & $19(23.4)$ \\
\hline $60-70$ & $10(12.3)$ \\
\hline $70-80$ & $8(9.8)$ \\
\hline $80-90$ & \\
\hline
\end{tabular} \\
\hline
\end{tabular}

Mediastinal lymphadenopathy was seen in 18 patients $(22.2 \%)$, and all of them had peri- and para-tracheal lymphadenopathy, 5 had aortopulmonary (AP) window lymphadenopathy and one patient had vascular lymphadenopathy. Pleural effusion was present in 12 patients (14.8\%). Pericardial effusion was less common and was only seen in 3 patients $(3.7 \%)$. Imaging findings are presented in Tables 2-4 in more detail.

Nine patients had follow-up CT scans on the third day after admission (based on clinical indications determined by infectious disease specialists). Out of those, 7 cases had an increase in radiologic grade, while 2 had no progression in radiologic staging.

Figures 1-4 demonstrate some of the selected imaging findings in this study.

\section{Discussion}

In this study we found that most patients infected with Sars-Cov-2 had significant findings in the CT scan. The most common findings were ground glass opacities, which were seen in 76 patients, septal thickening in 29 patients, subpleural line in 28 patients, crazy paving and air bronchogram in 15 patients. The least common findings were halo sign, tree in bud appearance, reverse halo sign and reticulonodular pattern. Most of the patients had bilateral involvement (66 patients) and only 11 had unilateral involvement. Mediastinal lymphadenopathy was seen in 18 patients. Three patients included in the study had a normal CT scan.

The COVID-19 epidemic is similar in some aspects to the previous coronavirus pandemics, such as SARS and

Table 2. Computed tomography radiologic staging of patients included in the study

Parameter
\begin{tabular}{|l|c|} 
Normal findings & $3(3.7)$ \\
\hline Stage 1 & $15(18.0)$ \\
\hline Stage 2 & $25(30.8)$ \\
\hline Stage 3 & $19(23.4)$ \\
\hline Stage 4 & $16(19.7)$ \\
\hline Stage 5 & $4(4.9)$ \\
\hline
\end{tabular}

Table 3. Pattern of lung involvement in patients with positive imaging findings

\begin{tabular}{|c|c|c|c|c|c|c|}
\hline Side & Unilateral & \multicolumn{2}{|c|}{$11(13.5 \%)$} & Bilateral & \multicolumn{2}{|c|}{$66(81.4 \%)$} \\
\hline \multirow{3}{*}{$\begin{array}{l}\text { Pattern } \\
\text { of involvement }\end{array}$} & Unifocal & $6(7.4 \%)$ & Multifocal & $61(75.3 \%)$ & Diffuse & $11(13.5 \%)$ \\
\hline & Peripheral & $69(85.1 \%)$ & Central & $15(18.5 \%)$ & Anterior & $11(13.5 \%)$ \\
\hline & Basal & $8(9.8 \%)$ & Posterior & $54(66.6 \%)$ & No involvement & $3(3.7 \%)$ \\
\hline \multirow[t]{2}{*}{ Involved lobes } & RUL & $57(70.3 \%)$ & RML & $57(70.3 \%)$ & RLL & $65(80.2 \%)$ \\
\hline & \multicolumn{3}{|c|}{ LLL } & $58(71.6 \%)$ & LUL & $58(71.6 \%)$ \\
\hline $\begin{array}{l}\text { Chest X-ray } \\
\text { involvement }\end{array}$ & Ground glass opacities & $24(29.6 \%)$ & Consolidation & $19(23.4 \%)$ & None & $38(46.9 \%)$ \\
\hline
\end{tabular}


Table 4. Specific radiologic findings in patients with lung involvement

\begin{tabular}{|c|c|c|c|}
\hline Radiologic findings & $n(\%)$ & Radiologic findings & $n(\%)$ \\
\hline Ground glass & $76(93.8)$ & Fibrotic changes & $6(7.4)$ \\
\hline Crazy paving & $15(18.5)$ & Bronchiectasis & $6(7.4)$ \\
\hline Airspace consolidation & $24(29.6)$ & Cavitation & $0(0.0)$ \\
\hline Septal thickening & $29(35.8)$ & Round pneumonia & $0(0.0)$ \\
\hline Nodular opacities & $10(12.3)$ & Air bronchogram & $15(18.5)$ \\
\hline Reticulonodular pattern & $3(3.7)$ & Subpleural line & $0(0.0)$ \\
\hline Atelectasis & $13(16.0)$ & Subpleural transparent line & $28(34.5)$ \\
\hline Tree in bud & $1(1.2)$ & Halo sign & $1(1.2)$ \\
\hline Peri-bronchovascular involvement & $13(16.0)$ & Reversed halo sign & $3(3.7)$ \\
\hline Emphysematous changes & $5(6.1)$ & None & $3(3.7)$ \\
\hline $\begin{array}{l}\text { Peri-tracheal and para-tracheal } \\
\text { lymphadenopathy }\end{array}$ & $18(22.2)$ & AP window lymphadenopathy & $5(6.1)$ \\
\hline PA thickening & $12(14.8)$ & Cardiomegaly & $9(11.1)$ \\
\hline Pleural effusion & $12(14.8)$ & Pericardial effusion & $3(3.7)$ \\
\hline
\end{tabular}

RUL - right upper lobe, RML - right middle lobe, RLL - right lower lobe, LLL - left lower lobe, LUL - left upper lobe.

MERS [14,15]. Studies done during the previous epidemics have shown that the most common imaging findings consisted of peripheral multifocal ground glass opacities and consolidations. Lymphadenopathies, cavitation and pneumothorax were not common findings. The experience with the previous epidemics, and the role of CT imaging in early diagnosis, prompted the use of this imaging method in the SARS-Co-V2 epidemic $[14,16]$.

One of the first reports of imaging findings in patients with COVID-19 was presented by Shi et al. This report was done on 81 patients who were all hospitalized in Chinese medical centers. They assigned patients to two groups, consisting of those with clinical and subclinical symptoms. The mean age of patients was 49.5 years. In this study the right lower lobe was the most frequent lung lobe involved, and patients without clinical symptoms had the fewest involved lobes, with a statistically significant difference between symptom-free patients and those with symptoms. Sixty-four patients (79\%) had bilateral lung involvement, 44 patients had peripheral involvement (54\%) and 36 patients had diffuse involvement (44\%). The least common findings were nodules (5 patients, $6 \%$ ), cystic changes (8 patients, $10 \%$ ), bronchiectasis ( 9 patients, $11 \%$ ), pleural effusion (4 patients, $5 \%$ ) and lymphadenopathy (5 patients, $6 \%$ ). Findings such as tree in bud appearance and cavitation and calcifications were not seen. Our findings were in accordance with Shi's study, with the difference that in almost all of our patients ground glass opacities were seen $(93.8 \%), 85.1 \%$ of patients had peripheral involvement, and $80.2 \%$ of patients had involvement in the right lower lobe, followed by the left lower lobe, left upper lobe, and right upper lobe. Lymphadenopathy and pleural effusion were less common $(22.2 \%$ and $14.8 \%$ respectively) compared to our other findings, but were still more common compared to Shi's study. These differences could be because of the indications for hospitalization of
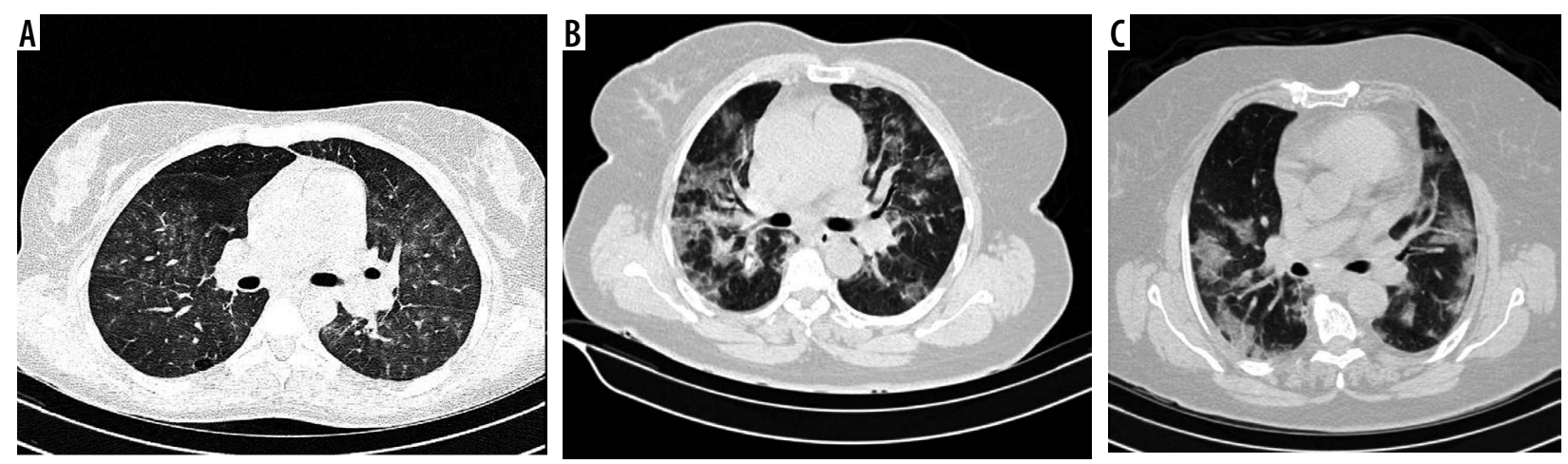

Figure 1. A) A 63-year-old woman presented to EM ward with cough and dyspnea. The chest computed tomography (CT) shows bilateral multifocal centrilobular ground glass opacities. B) A 71-year-old woman presented with dyspnea. The chest CT shows bilateral multiple ground glass opacities with interlobular septal thickening more prominently at peripheral and posterior regions of both lungs. C) A 39-year-old male patient with multifocal patchy groundless opacities; note the fine subpleural transparent region between the opacities and pleura 

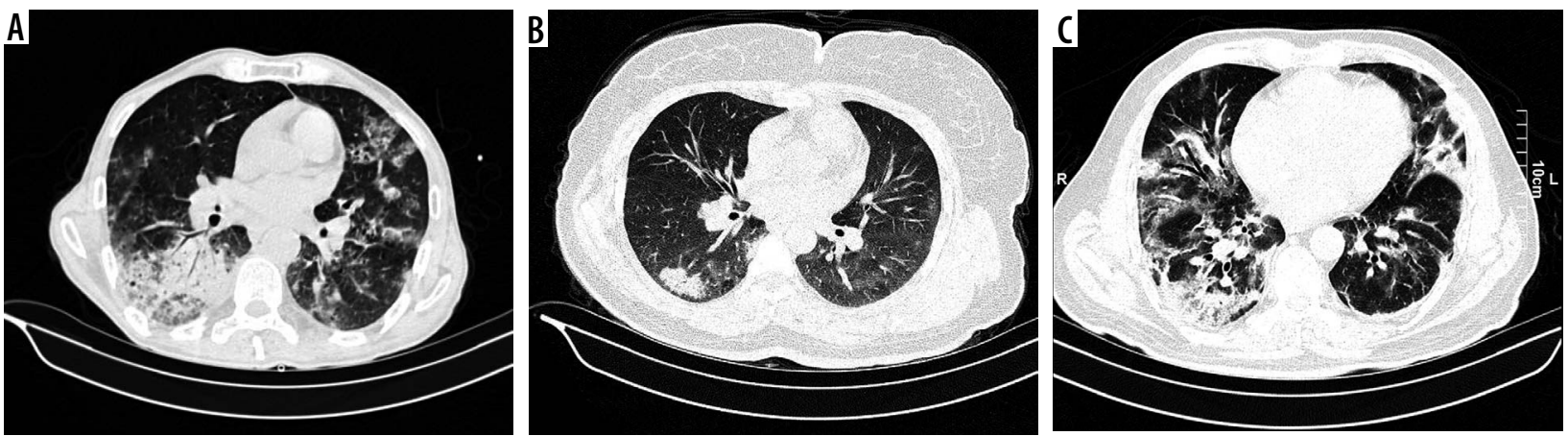

Figure 2. A) A 54-year-old man. Chest computed tomography (CT) shows both airspace consolidation with air bronchogram at right side and multiple airspace ground glass opacities. B) A 49-year-old woman. Chest CT shows bilateral fine ground glass opacities and transparent subpleural sign and airspace consolidation at the posterior. margin right lung. C) A 61-year-old man. (T showed multiple airspace opacities with bronchiectasis at the right side
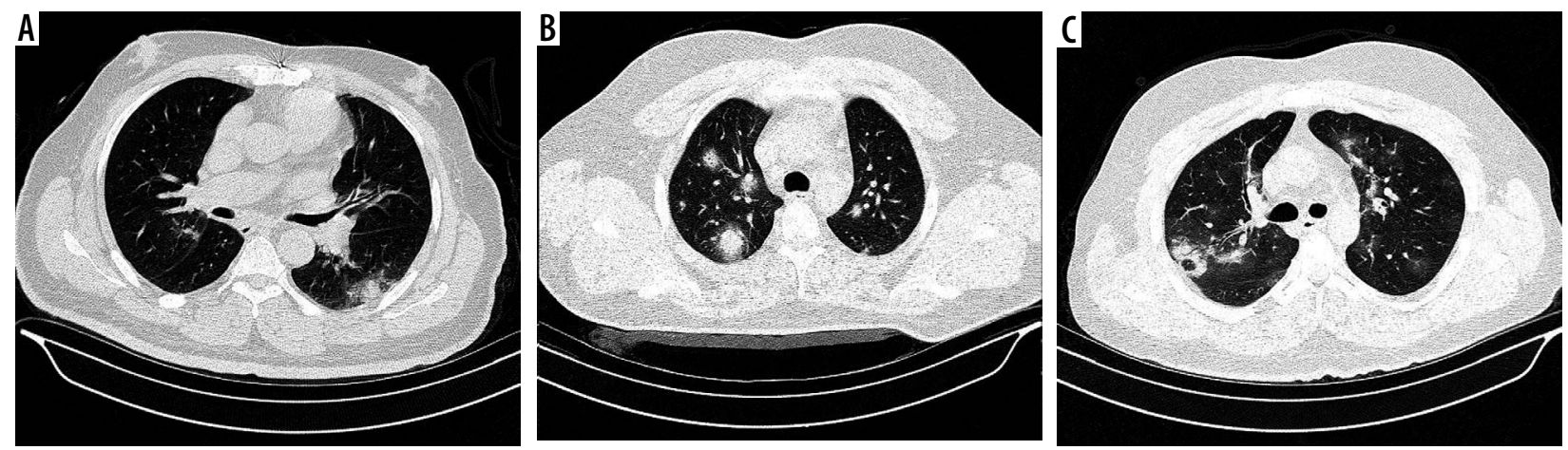

Figure 3. A) A 41-year-old man. the chest computed tomography (CT) showed nodular opacities surrounded with ground glass opacities at left lower lobe which is known as halo sign. B) A 53-year-old patient with multiple nodular opacities with halo sign. C) A43-year-old patient. (hest (T shows ground-glass opacities bilaterally and reversed halo sign at the right upper lobe
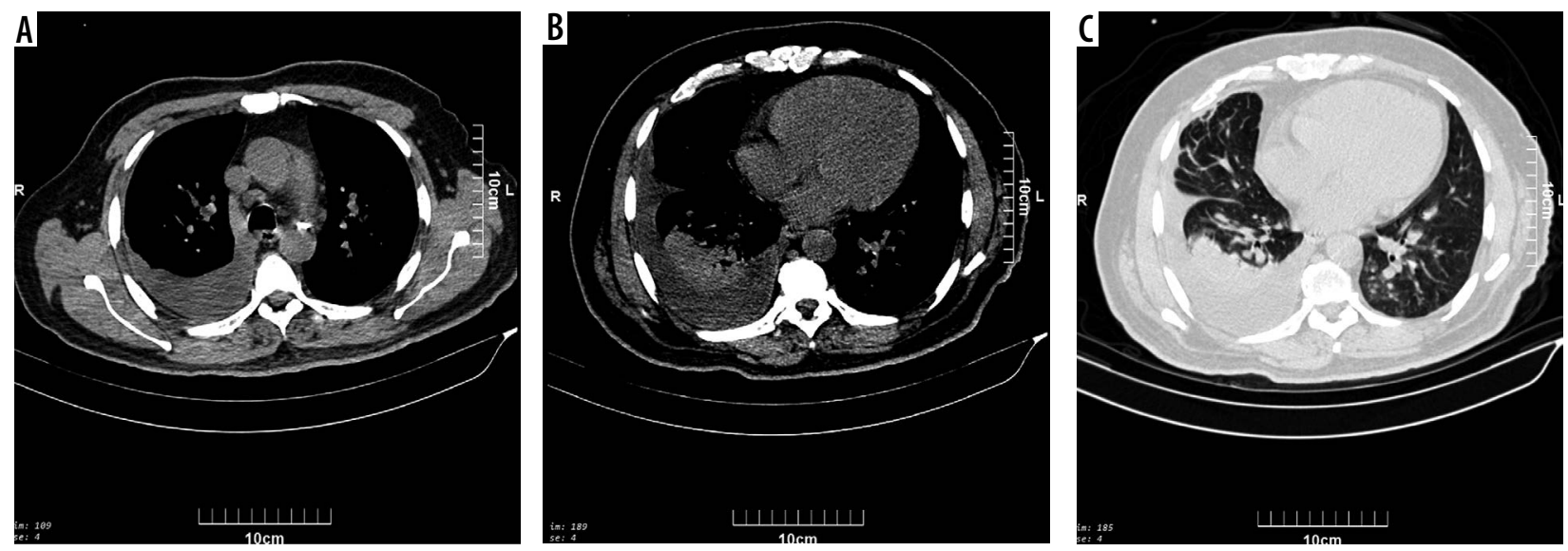

Figure 4. All three images $(A, B, C)$ belong to a 62-year-old man. Airspace consolidation with air bronchogram is seen at the right lower lobe and ground glass opacities at left lower lobe which are associated with right pleural effusion and pre-tracheal lymph node enlargement. This patient had a severe degree of effusion, not routinely witnessed with viral pneumonia

patients. In Shi's study a considerable number of patients with no clinical symptoms or mild symptoms were hospitalized, while in our study only those with moderate to severe symptoms were hospitalized. Furthermore, in the early phases of the epidemic case finding was actively being done in China, and thus patients were hospitalized in early stages, while in our situation, most patients were diagnosed after being symptomatic and being referred to a tertiary care center. This could have caused the increase in number of positive imaging findings [17]. Further evidence in this regard could be suggested by the fact that most of the atypical and less common findings were seen in those with higher stages (stages 3-4).

Another early study performed in South Korea focused on imaging findings of COVID-19. South Korea was one of the countries which was hit hard by the virus, but was able to reduce the spread of the disease by active case finding and wide scale testing. In the study done by Yoon et al. nine patients with proven COVID-19 were included. Eight patients had bilateral lung involvement, and the median number of involved lobes was five. The most frequently involved lobes were the right lower lobe and left upper lobe. 
These results were in accordance with our results, and the extent of involvement was similar to our study. This could be because of late detection and hospitalization of these patients in the early stages of the epidemic [18]. Liu et al. conducted a study on Chinese patients infected with the virus. They classified the patients in 4 groups based on disease severity, and compared radiologic findings in each of the groups. They found that out of 6 patients with mild symptoms, 3 had normal CT images, while all those with moderate symptoms had abnormalities in their imaging, the most common being ground glass opacities. Other common findings were paving stone sign, inter and intralobular septal thickening and air bronchogram. In 21 patients with severe symptoms, ground glass opacities and consolidations were the most significant findings [19].

Later in the course of the epidemic, more studies were published from China, this time focusing on early findings of chest CTs. A study done by Ng et al. included CT images as early as 1 day after symptoms appeared with the median time being 3 days. They found that out of 21 cases, 2 had normal CT scans, 18 patients had ground glass opacities (86\%), and 13 patients had air space consolidation (62\%). Of all the 18 patients with abnormal CT imaging, 11 had dominant ground glass lesions, four had dominant airspace consolidation and four had mixed involvement. Lower lobes of the lungs were more commonly involved than the upper lobes (16 vs. 11 respectively). No cases of pleural effusions, lymphadenopathy, cavitation or pleural effusion were seen [20]. The major findings of this study supported our conclusions, except the fact that we observed the aforementioned findings not seen in this study. This could be because of the fact that our patients were hospitalized in late stages of the disease, mostly because of the fact that the condition was not diagnosed because of a lack of wide scale testing. Furthermore, we included more patients, which could result in less common findings being seen.

In the present study we also examined chest X-rays belonging to patients. Most of the cases with positive findings suggestive of SARS-Cov-2 infection were those in higher stages (particularly stages 3-4), while those in stage 1 and 2 had lower numbers of positive findings.
The overall sensitivity was estimated to be $53 \%$. The previous conclusions could be applicable here as well, which could justify the normal chest X-rays in early stages.

Overall it seems that radiologic findings of COVID-19 are sensitive enough to enable a rapid diagnosis amid an epidemic (most importantly in countries with limited access to molecular testing). Challenges still remain regarding the appropriate interpretation of imaging findings with respect to time of involvement, and the step by step alterations made in imaging findings. In this study, we included 81 patients who were hospitalized because of moderate to severe symptoms, and we did not include asymptomatic patients, or those with mild symptoms, who may not have sought medical attention in the first place. Our study was performed on patients presenting a single center, and large scale studies may show different findings.

\section{Conclusions}

The sensitivity of CT imaging in diagnosing COVID-19 was $96 \%$. The most common finding in imaging was bilateral, multifocal ground glass opacities, with other findings such as air bronchogram, consolidation and septal thickening being also frequently reported. Furthermore, other non-common imaging findings were witnessed with an increased rate compared to similar studies. The most common lobe involved was the right lower lobe, and the most common region of involvement was posterior and peripheral regions of the lungs. Nine patients had CT imaging done one week after hospitalization due to follow-up, and 7 of these had an increase in stage of involvement, while 2 did not show any significant difference. We also found that chest X-rays had a sensitivity of $53.0 \%$, with ground glass opacities being more common than consolidations. Overall, we report a higher percentage of specific radiologic findings, which we attribute to our patients being those hospitalized because of moderate to severe symptoms.

\section{Conflict of interest}

The authors report no conflict of interest.

\section{References}

1. Li X, Geng M, Peng Y, et al. Molecular immune pathogenesis and diagnosis of COVID-19. J Pharm Anal 2020; 10: 102-108.

2. Sun J, He WT, Wang L, et al. COVID-19: epidemiology, evolution, and cross-disciplinary perspectives. Trends Mol Med 2020; 26: $483-$ 495.

3. Fehr AR, Perlman S. Coronaviruses: an overview of their replication and pathogenesis. Methods Mol Biol 2015; 1282: 1-23.

4. Ye F, Xu S, Rong Z, et al. Delivery of infection from asymptomatic carriers of COVID-19 in a familial cluster. Int J Infect Dis 2020; 94: 133-138.
5. Pung R, Chiew CJ, Young BE, et al. Investigation of three clusters of COVID-19 in Singapore: implications for surveillance and response measures. Lancet 2020; 395: 1039-1046.

6. Esposito A, Palmisano A, Scotti GM, et al. Why is chest CT important for early diagnosis of COVID-19? Prevalence matters. medRxiv 2020. doi: https://doi.org/10.1101/2020.03.30.20047985.

7. Fang Y, Zhang H, Xie J, et al. Sensitivity of chest CT for COVID-19: comparison to RT-PCR. Radiology 2020; 296: E115-E117.

8. World Health Organization. Clinical management of severe acute respiratory infection when novel coronavirus ( $\mathrm{nCoV}$ ) infection is 
suspected: interim guidance, 25 January 2020. Iris 2020. Available from: https://apps.who.int/iris/handle/10665/330854.

9. Kanne JP. Chest CT findings in 2019 novel coronavirus (2019-nCoV) infections from Wuhan, China: key points for the radiologist. Radiology 2020; 295: 16-17.

10. Kanne JP, Little BP, Chung JH, et al. Essentials for radiologists on COVID-19: an update - radiology scientific expert panel. Radiology 2020; 296: E113-E114.

11. Mahdavi A, Khalili N, Davarpanah AH, et al. Radiologic management of COVID-19: preliminary experience of the Iranian Society of Radiology COVID-19 Consultant Group (ISRCC). Iran J Radiol 2020; 17: e102324.

12. Jin $\mathrm{Y}-\mathrm{H}$, Cai L, Cheng Z-S, et al. A rapid advice guideline for the diagnosis and treatment of 2019 novel coronavirus (2019-nCoV) infected pneumonia (standard version). Mil Med Res 2020; 7: 4.

13. World Health Organization. Diagnostic detection of Wuhan coronavirus 2019 by real-time RT-PCR. 2020.

14. Ahmadzadeh J, Mobaraki K, Mousavi SJ, et al. The risk factors associated with MERS-CoV patient fatality: a global survey. Diagn Microbiol Infect Dis 2020; 96: 114876

15. Aghazadeh-Attari J, Mohebbi I, Mansorian B, et al. Epidemiological factors and worldwide pattern of Middle East respiratory syndrome coronavirus from 2013 to 2016. Int J Gen Med 2018; 11:121-125.

16. Hosseiny M, Kooraki S, Gholamrezanezhad A, et al. Radiology perspective of coronavirus disease 2019 (COVID-19): lessons from severe acute respiratory syndrome and middle east respiratory syndrome. AJR Am J Roentgenol 2020; 214: 1078-1082.

17. Shi H, Han X, Jiang N, et al. Radiological findings from 81 patients with COVID-19 pneumonia in Wuhan, China: a descriptive study. Lancet Infect Dis 2020; 20: 425-434.

18. Yoon SH, Lee KH, Kim JY, et al. Chest radiographic and CT findings of the 2019 novel coronavirus disease (COVID-19): analysis of nine patients treated in Korea. Korean J Radiol 2020; 21: 494-500.

19. Liu KC, Xu P, Lv WF, et al. CT manifestations of coronavirus disease-2019: a retrospective analysis of 73 cases by disease severity. Eur J Radiol 2020; 126: 108941.

20. Ng M-Y, Lee EY, Yang J, et al. Imaging profile of the COVID-19 infection: radiologic findings and literature review. Radiol Cardiothorac Imag 2020; 2: e200034 